Journal of

Molecular Microbiology

and Biotechnology
Published online: September 29, 2008

\title{
A Genomic View on Nitrogen Metabolism and Nitrogen Control in Mycobacteria
}

\author{
Johannes Amon Fritz Titgemeyer Andreas Burkovski \\ Lehrstuhl für Mikrobiologie, Friedrich-Alexander-Universität Erlangen-Nürnberg, Erlangen, Deutschland
}

\author{
Key Words \\ Ammonium • Mycobacterium • Glutamine synthetase • \\ Nitrogen control $\cdot$ Nitrogen regulation $\cdot G \ln R$
}

\begin{abstract}
Knowledge about nitrogen metabolism and control in the genus Mycobacterium is sparse, especially compared to the state of knowledge in related actinomycetes like Streptomyces coelicolor or the close relative Corynebacterium glutamicum. Therefore, we screened the published genome sequences of Mycobacterium smegmatis, Mycobacterium tuberculosis, Mycobacterium bovis, Mycobacterium avium ssp. paratuberculosis and Mycobacterium leprae for genes encoding proteins for uptake of nitrogen sources, nitrogen assimilation and nitrogen control systems, resulting in a detailed comparative genomic analysis of nitrogen metabolism-related genes for all completely sequenced members of the genus. Transporters for ammonium, nitrate, and urea could be identified, as well as enzymes crucial for assimilation of these nitrogen sources, i.e. glutamine synthetase, glutamate dehydrogenase, glutamate synthase, nitrate reductase, nitrite reductase, and urease proteins. A reduction of genes encoding proteins for nitrogen transport and metabolism was observed for the pathogenic mycobacteria, especially for M. leprae. Signal transduction components identified for the different species include adenylyl- and uridylyltransferase and a $\mathrm{P}_{\| I}$-type signal transduction protein. Exclusively for M. smegmatis, two homologs of putative nitrogen regulato-
\end{abstract}

ry proteins were found, namely GInR and AmtR, while in other mycobacteria, AmtR was absent and GInR seems to be the nitrogen transcription regulator protein.

Copyright $\odot 2008$ S. Karger AG, Basel

\section{Introduction}

The genus Mycobacterium includes saprophytic soil bacteria like Mycobacterium smegmatis as well as human pathogens such as Mycobacterium leprae and, the most prominent member, Mycobacterium tuberculosis. The latter is responsible for an estimated eight million new infections and nearly two million deaths each year [Dye et al., 2005]. Due to the ability of M. tuberculosis to replicate within macrophages, these bacteria are able to escape the human immune system and can persist in the human lung for decades in a state of dormancy prior to reactivation. Medical treatment of tuberculosis is further impeded by the appearance of strains, which are highly resistant against almost all standard antibiotics. For the design of new drugs, a more thorough understanding of the molecular biology and physiology of mycobacteria becomes crucial. A milestone in this respect has been achieved by sequencing several mycobacterial genomes including M. tuberculosis, M. leprae, Mycobacterium avium and Mycobacterium bovis [Cole et al., 1998, 2001; Garnier et al., 2003; Li et al., 2005]. This is also the case for the fastgrowing M. smegmatis (http://www.tigr.org/), which has

\section{KARGER \\ Fax +41613061234 \\ E-Mail karger@karger.ch}

www.karger.com
(C) 2008 S. Karger AG, Basel

$1464-1801 / 09 / 0171-0020 \$ 26.00 / 0$

Accessible online at:

www.karger.com $/ \mathrm{mmb}$
Andreas Burkovski

Lehrstuhl für Mikrobiologie, Friedrich-Alexander-Universität Erlangen-Nürnberg

Staudtstrasse 5, DE-91058 Erlangen (Germany)

Tel. +49 913185 28086, Fax +49 91318528082

E-Mail aburkov@biologie.uni-erlangen.de 
been used as the basis of mycobacterial genetics [Pelicic et al., 1998] and as a non-pathogen substitute to study the metabolic and regulatory pathways of $M$. tuberculosis [Bange et al., 1999; Titgemeyer et al., 2007].

We are especially interested in nitrogen metabolism and regulation in mycolic acids-containing actinomycetes. Since almost all of the macromolecules in a bacterial cell, e.g. proteins, nucleic acids and cell wall components, contain nitrogen, prokaryotes have developed elaborate mechanisms to provide an optimal nitrogen supply for cell maintenance and growth, and to overcome and survive situations of nitrogen starvation. In actinomycetes, the uptake and assimilation of nitrogen sources has been studied intensively for the close relatives of mycobacteria Corynebacterium glutamicum [for reviews, see Burkovski, 2003a, b, 2005, 2007; Hänßler and Burkovski, 2008] and Streptomyces coelicolor [Reuther and Wohlleben, 2007], while for the genus Mycobacterium, work concentrated mainly on the glutamine synthetase GlnA1 and its adenylylase $\mathrm{GlnE}$ as potential drug targets [Carroll et al., 2008; Harth et al., 2005; Hotter et al., 2008; Nordqvist et al., 2008]. Using the information available for these well-studied model organisms, we compiled a list of nitrogen metabolism-related genes based on in silico analyses of the published genome sequence of $M$. smegmatis $\mathrm{mc}^{2} 155$ and their respective homologs in various pathogenic mycobacteria to provide comprehensive information for further experimental studies.

\section{Results and Discussion}

\section{Genes Related to Ammonium Uptake and}

\section{Assimilation}

Ammonium is the preferred nitrogen source of most microorganisms and consequently ammonium uptake systems and assimilating enzymes are widely distributed among bacteria. A highly conserved apparent operon was found in all mycobacterial genomes comprising the genes $a m t B-g \ln K-g \ln D$. The $a m t B$ gene encodes a putative ammonium uptake system, which has been functionally characterized in enterobacteria, Bacillus subtilis [Detsch and Stülke, 2003] and C. glutamicum [Jakoby et al., 1999; Meier-Wagner et al., 2001; Walter et al., 2008]. The two other genes are crucial for nitrogen-dependent signal transduction. The $g \ln K$ gene is coding for a $\mathrm{P}_{\mathrm{II}}$-type signal transduction protein and $g \ln D$ encodes a GlnK modifying/demodifying enzyme, which works as a uridylyltransferase in enterobacteria and acts as an adenylyltransferase in S. coelicolor [Hesketh et al., 2002] and C. glutamicum [Strösser et al., 2004]. In accordance with the massive gene decay observed in the leprosy bacillus, in $M$. leprae all genes of this operon are pseudogenes and carry mutations, which prevent synthesis of active proteins. For example, amtB exhibits a mutated start codon, thus preventing translation, and multiple frame shifts resulting in a nonfunctional reading frame.

In M. smegmatis, another two genes, designated amt $A$ (msmeg_4635) and amt1 (msmeg_6259) were found, which encode additional putative ammonium transporters. While for amtA no apparent operon was observed, the latter one is organized in a cluster together with genes that apparently encode different functional domains of a class III glutamine synthetase (msmeg_6260), a class II glutamine amidotransferase (msmeg_6261), and domains of a glutamate synthase (msmeg_6262, msmeg_6263). This conserved region seems to be of Pseudomonadales origin according to the BLAST results and is in the sequenced actinomycetes only found in the genomes of $M$. smegmatis, Nocardia farcinica, and Rhodococcus sp.

Once ammonium has entered the cell via diffusion across the cytoplasmic membrane or by protein-dependent transport, this nitrogen source has to be assimilated. Most bacteria have two primary pathways to facilitate the incorporation of ammonium into the key nitrogen donors for biosynthetic reactions, L-glutamate and L-glutamine, namely the glutamate dehydrogenase (GDH) and the glutamine synthetase/glutamate synthase pathways (GS/GOGAT). Assimilation via glutamate dehydrogenase is bioenergetically more favorable, as the GS/GOGAT pathway utilizes an additional ATP per assimilated ammonium. Consequently, GDH is often preferentially used in ammonium-rich medium. However, when the cells face nitrogen limitation, assimilation via GDH is not sufficient due to the low affinity of the enzyme and the GS/GOGAT pathway is recruited for ammonium assimilation. Interestingly, only the genome of $M$. smegmatis features a $g d h A$ gene coding for an assimilatory NADPHdependent glutamate dehydrogenase. This gene is missing in the other four mycobacterial species investigated here and consequently ammonium assimilation depends on the GS/GOGAT pathway in these bacteria.

The genes $g \ln A 1$ and $g \ln A 2$ are found in all mycobacterial genomes in a conserved region together with $g \ln E$, encoding an adenylyltransferase that is essential in $M$. tuberculosis [Parish et al., 2000] and was shown to regulate glutamine synthetase activity [Carroll et al., 2008]. Homologs of the GlnA3 and GlnA4 proteins were found in all mycobacterial genomes except in M. leprae, while for GlnA4 at least three homologous proteins were iden- 
tified in the genome of $M$. smegmatis via BLASTP (MSMEG_2595, MSMEG_3828, MSMEG_1116), of which MSMEG_2595 shares the highest identity to the $S$. coelicolor GlnA4 protein (62\%, SCO1613 [Rexer et al., 2006]). Besides homologs of $g \ln A 1, g \ln A 2, g \ln A 3$ and glnA4, M. smegmatis possesses various additional genes that seem to encode homologs of glutamine synthetases (see below and table 1), and which are missing in other mycobacterial genomes. MSMEG_5374 shares with 51\% the highest identity to glutamine synthetases-like proteins in the soil-dwelling $\alpha$ proteobacteria Rhodopseudomonas palustris CGA009 (RPA0984) and Bradyrhizobium japonicum USDA 110 (BLL1069). Furthermore, msmeg_5374 features with 63\% a GC-content significantly below the average $67 \% \mathrm{G}+\mathrm{C}$ content of the genome of M. smegmatis. For MSMEG_3827, only weak identities (about 30\%) to various not further characterized glutamine synthetase-like proteins in proteobacteria were found. MSMEG_6260, which also shares 69\% identity to a putativeglutaminesynthetase of N. farcinica(NFA21040), seems to be a type III glutamine synthetase with around $45 \%$ identity to GS III enzymes of various Synechococcus and Pseudomonas species, also indicated by the BLASTP results of the corresponding putative operon ( $\mathrm{msmeg}_{-}$ 6260-6264). Another putative type III GS might be encoded by msmeg_6693, exhibiting 35-45\% identical amino acids to various rhizobial GS III enzymes and $33 \%$ identity to the glutamine synthetase III of Agrobacterium tumefaciens (Atu4230). While it was already shown for M. tuberculosis that only GlnA1 is abundantly expressed and essential for bacterial homeostasis [Harth et al., 2005], the physiological role and function of the various other glutamine synthetase-encoding genes, especially in the genome of $M$. smegmatis, remain to be verified.

In all mycobacterial genomes, a highly conserved apparent operon encoding the large and small subunit of the glutamate synthase (GOGAT; gltB and gltD) was observed. In addition, the genome of $M$. smegmatis features several additional copies of $g l t B$ (msmeg_5594, msmeg 6263, msmeg6459) and gltD (msmeg_6262, msmeg_6458) that are not found in other mycobacteria.

\section{Genes Encoding Transporters of Alternative Nitrogen}

Sources and Assimilatory Enzymes

Ammonium cannot only be provided directly, but also generated from alternative nitrogen sources. A prominent example is urea, which is degraded to ammonium and carbon dioxide by ureases. For M. smegmatis, two putative urease-encoding operons were found, of which only one (msmeg_3623-3627) exhibits homology to the ure gene clusters in M. tuberculosis and M. bovis based on gene identity and arrangement. The second one exhibits striking similarities to the urease subunits - encoding genes from $\alpha$ proteobacteria (e.g. Pseudomonas syringae, Helicobacter pylori, and various Burkholderia species) with DNA sequence identities between 60 and $70 \%$, an identical operon arrangement and a $u r e A B$ fusion gene. No urease operon or urease-related genes were found in $M$. avium and M. leprae. Also, M. smegmatis is the only mycobacterial species to feature a distinct operon ( $m s$ meg_2978-2982) encoding the subunits of a putative urea $\mathrm{ABC}$ transporter, emphasizing - together with the presence of the different urease-encoding operons - the importance of urea as a nitrogen source for this species.

Furthermore, we found genes encoding the necessary components for the complete reduction of nitrate to ammonium via nitrate reductase (NarGHJI, NarX) and nitrite reductase (NirBD), including putative nitrite/nitrate transporters (homologs of Escherichia coli NarK and $\mathrm{NarU}$ ), in the genomes of all screened mycobacteria except $M$. leprae, which seems to possess a nitrate/nitrite antiporter (ML0844) but is missing genes encoding the corresponding enzymes for reduction and assimilation.

\section{Signal Transduction}

Among mycobacteria, proteins involved in signalling and posttranslational modification are best characterized in M. tuberculosis [Parish and Stoker, 2000]. Since glutamine synthetase in M. tuberculosis is essential for this bacterium and consequently an important drug target [Harth et al., 1994, 2005; Nordqvist et al., 2008; Tullius et al., 2003], work concentrated first on the $g \ln E$ gene product adenylyltransferase, which is involved in posttranslational modification and regulation of this enzyme. It was shown that $g \ln E$ is essential in this organism [Parish and Stoker, 2000], an observation that is in agreement with the crucial function of adenylyltransferase in regulation of GS activity [Carroll et al., 2008]; furthermore, the $M$. tuberculosis glnE promoter is upregulated in ammonia- or glutamine-containing media, at least in the heterologous host M. smegmatis [Pashley et al., 2006], while the transcriptional organization of the $g \ln A 1-g \ln E$ $g \ln A 2$ gene cluster remains to be elucidated further [Hotter et al., 2008].

GlnD seems to have no crucial function in the regulation of ammonium assimilation in $M$. tuberculosis [Read et al., 2007]. This is in agreement with observations made in S. coelicolor and C. glutamicum [Hesketh et al., 2002; Strösser et al., 2004]. In contrast to the signal transfer via $\mathrm{GlnD}, \mathrm{G} \operatorname{lnK}$ and $\mathrm{GlnE}$ to GS as shown in E. coli, enzyme 
Table 1. Nitrogen metabolism-related genes in mycobacteria: the given references were selected to provide information for representative, well-studied homologs

\begin{tabular}{|c|c|c|c|c|c|c|c|}
\hline Enzyme/function & $\begin{array}{l}\text { Gene } \\
\text { name }\end{array}$ & $\begin{array}{l}\text { M. smegmatis } \\
\mathrm{mc}^{2} 155\end{array}$ & $\begin{array}{l}\text { M. tuber- } \\
\text { culosis } \\
\text { H37rv }\end{array}$ & $\begin{array}{l}\text { M. leprae } \\
\text { TN }\end{array}$ & $\begin{array}{l}\text { M. avium } \\
\text { ssp. para- } \\
\text { tuberculosis }\end{array}$ & $\begin{array}{l}\text { M. bovis } \\
\text { ssp. bovis } \\
\text { AF2122/97 }\end{array}$ & Reference/nearest homologs \\
\hline $\begin{array}{l}\text { Assimilatory nitrite } \\
\text { reductase }\end{array}$ & $\begin{array}{l}\operatorname{nir} B \\
\operatorname{nirD}\end{array}$ & $\begin{array}{l}\text { msmeg_0427 } \\
\text { msmeg_0428 }\end{array}$ & $\begin{array}{l}R v 0252 \\
R v 0253\end{array}$ & $\begin{array}{l}\mathrm{n} / \mathrm{a} \\
\mathrm{n} / \mathrm{a}\end{array}$ & $\begin{array}{l}\text { MAP_3702 } \\
\text { MAP_3703 }\end{array}$ & $\begin{array}{l}M b 0258 \\
M b 0259\end{array}$ & $\begin{array}{l}\text { E. coli nitrite reductase operon } \\
\text { [Wang and Gunsalus, 2000] }\end{array}$ \\
\hline Hydrolysis of urea & $\begin{array}{l}\text { ureE } \\
\text { ureF } \\
\text { ureAB } \\
\text { ureC } \\
\text { ure } G \\
\text { ureD }\end{array}$ & $\begin{array}{l}\text { msmeg_1091 } \\
\text { msmeg_1092 } \\
\text { msmeg_1093 } \\
\text { msmeg_1094 } \\
\text { msmeg_1095 } \\
\text { msmeg_1096 }\end{array}$ & $\begin{array}{l}\mathrm{n} / \mathrm{a} \\
\mathrm{n} / \mathrm{a} \\
\mathrm{n} / \mathrm{a} \\
\mathrm{n} / \mathrm{a} \\
\mathrm{n} / \mathrm{a} \\
\mathrm{n} / \mathrm{a}\end{array}$ & $\begin{array}{l}\mathrm{n} / \mathrm{a} \\
\mathrm{n} / \mathrm{a} \\
\mathrm{n} / \mathrm{a} \\
\mathrm{n} / \mathrm{a} \\
\mathrm{n} / \mathrm{a} \\
\mathrm{n} / \mathrm{a}\end{array}$ & $\begin{array}{l}\mathrm{n} / \mathrm{a} \\
\mathrm{n} / \mathrm{a} \\
\mathrm{n} / \mathrm{a} \\
\mathrm{n} / \mathrm{a} \\
\mathrm{n} / \mathrm{a} \\
\mathrm{n} / \mathrm{a}\end{array}$ & $\begin{array}{l}\mathrm{n} / \mathrm{a} \\
\mathrm{n} / \mathrm{a} \\
\mathrm{n} / \mathrm{a} \\
\mathrm{n} / \mathrm{a} \\
\mathrm{n} / \mathrm{a} \\
\mathrm{n} / \mathrm{a}\end{array}$ & $\begin{array}{l}\text { Pseudomonas syringae urease operon } \\
\text { (psyr_2200-2195) }\end{array}$ \\
\hline Unknown & $g \ln A^{*}$ & msmeg_1116 & $\mathrm{n} / \mathrm{a}$ & $\mathrm{n} / \mathrm{a}$ & $\mathrm{n} / \mathrm{a}$ & $\mathrm{n} / \mathrm{a}$ & [Harth et al., 2005] \\
\hline Ammonium uptake & $a m t B$ & msmeg_2425 & $R v 2920 c$ & (ML1627) & $M A P \_2988 c$ & $M b 2944 c$ & amtB-glnK-glnD S. coelicolor \\
\hline Signal transduction & $g \ln K$ & msmeg_2426 & $R v 2919 c$ & $(M L 1626)$ & MAP_2987c & $M b 2943 c$ & [Fink et al., 2002] \\
\hline $\begin{array}{l}\text { Post-translational } \\
\text { regulation of } \mathrm{GlnK}\end{array}$ & $g \ln D$ & msmeg_2427 & $R v 2918 c$ & (ML1625) & $M A P \_2986 c$ & $M b 2942 c$ & \\
\hline Unknown & $g \ln A 4$ & msmeg_2595 & $R v 2860 c$ & $n / a$ & MAP_2931c & $M b 2885 c$ & [Harth et al., 2005] \\
\hline Urea uptake & $\begin{array}{l}\text { urtE } \\
\text { urtD } \\
\text { urtC } \\
\text { urtB } \\
\text { urtA }\end{array}$ & $\begin{array}{l}\text { msmeg_2978 } \\
\text { msmeg_2979 } \\
\text { msmeg_2980 } \\
\text { msmeg_2981 } \\
\text { msmeg_2982 }\end{array}$ & $\begin{array}{l}\mathrm{n} / \mathrm{a} \\
\mathrm{n} / \mathrm{a} \\
\mathrm{n} / \mathrm{a} \\
\mathrm{n} / \mathrm{a} \\
\mathrm{n} / \mathrm{a}\end{array}$ & $\begin{array}{l}\mathrm{n} / \mathrm{a} \\
\mathrm{n} / \mathrm{a} \\
\mathrm{n} / \mathrm{a} \\
\mathrm{n} / \mathrm{a} \\
\mathrm{n} / \mathrm{a}\end{array}$ & $\begin{array}{l}\mathrm{n} / \mathrm{a} \\
\mathrm{n} / \mathrm{a} \\
\mathrm{n} / \mathrm{a} \\
\mathrm{n} / \mathrm{a} \\
\mathrm{n} / \mathrm{a}\end{array}$ & $\begin{array}{l}\mathrm{n} / \mathrm{a} \\
\mathrm{n} / \mathrm{a} \\
\mathrm{n} / \mathrm{a} \\
\mathrm{n} / \mathrm{a} \\
\mathrm{n} / \mathrm{a}\end{array}$ & $\begin{array}{l}\text { C. glutamicum urease transport operon } \\
\text { [Beckers et al., 2004] }\end{array}$ \\
\hline Ammonium assimilaton & $\begin{array}{l}\text { gltB } \\
\text { gltD }\end{array}$ & $\begin{array}{l}\text { msmeg_3225 } \\
\text { msmeg_3226 }\end{array}$ & $\begin{array}{l}R v 3859 \\
R v 3858\end{array}$ & $\begin{array}{l}\text { ML0061 } \\
\text { ML0062 }\end{array}$ & $\begin{array}{l}\text { MAP_0172 } \\
\text { MAP_0173 }\end{array}$ & $\begin{array}{l}M b 3888 c \\
M b 3889 c\end{array}$ & $\begin{array}{l}\text { C. glutamicum glutamate synthase } \\
\text { [Beckers et al., 2001; Schulz et al., 2001] }\end{array}$ \\
\hline Unknown & $g \ln A 3$ & msmeg_3561 & $R v 1878$ & $n / a$ & MAP_1599 & $M b 1910$ & [Harth et al., 2005] \\
\hline Hydrolysis of urea & $\begin{array}{l}\text { ureD } \\
\text { ureG } \\
\text { ureF } \\
\text { ureC } \\
\text { ureB } \\
\text { ureA }\end{array}$ & $\begin{array}{l}n / a \\
\text { msmeg_3623 } \\
\text { msmeg_3624 } \\
\text { msmeg_3625 } \\
\text { msmeg_3626 } \\
\text { msmeg_3627 }\end{array}$ & $\begin{array}{l}R v 1853 \\
R v 1852 \\
R v 1851 \\
R v 1850 \\
R v 1849 \\
R v 1848\end{array}$ & $\begin{array}{l}\mathrm{n} / \mathrm{a} \\
\mathrm{n} / \mathrm{a} \\
\mathrm{n} / \mathrm{a} \\
\mathrm{n} / \mathrm{a} \\
\mathrm{n} / \mathrm{a} \\
\mathrm{n} / \mathrm{a}\end{array}$ & $\begin{array}{l}\mathrm{n} / \mathrm{a} \\
\mathrm{n} / \mathrm{a} \\
\mathrm{n} / \mathrm{a} \\
\mathrm{n} / \mathrm{a} \\
\mathrm{n} / \mathrm{a} \\
\mathrm{n} / \mathrm{a}\end{array}$ & $\begin{array}{l}M b 1884 \\
M b 1883 \\
M b 1882 \\
M b 1881 \\
M b 1880 \\
M b 1879\end{array}$ & $\begin{array}{l}\text { M. tuberculosis urease operon } \\
\text { [Clemens et al., 1995] }\end{array}$ \\
\hline Unknown & $g \ln A^{*}$ & msmeg_3827 & $\mathrm{n} / \mathrm{a}$ & $\mathrm{n} / \mathrm{a}$ & $\mathrm{n} / \mathrm{a}$ & $\mathrm{n} / \mathrm{a}$ & $\begin{array}{l}\text { E. coli K12 putative glutamine synthetase } \\
\text { (b1297) }\end{array}$ \\
\hline Unknown & $g \ln A^{*}$ & msmeg_3828 & $\mathrm{n} / \mathrm{a}$ & $\mathrm{n} / \mathrm{a}$ & $\mathrm{n} / \mathrm{a}$ & $\mathrm{n} / \mathrm{a}$ & [Harth et al., 2005] \\
\hline Ammonium assimilation & $g \ln A 1$ & msmeg_4290 & $R v 2220$ & ML0925 & MAP_1962 & $M b 2244$ & [Harth et al., 2005] \\
\hline $\begin{array}{l}\text { Post-translational } \\
\text { regulation of GSI }\end{array}$ & $g \ln E$ & msmeg_4293 & $R v 2221 c$ & ML1630 & MAP_1965c & $M b 2245 c$ & [Carroll et al., 2008] \\
\hline Unknown & $g \ln A 2$ & msmeg_4294 & $R v 2222 c$ & ML1631 & $M A P_{-} 1966 c$ & $M b 2246 c$ & [Harth et al., 2005] \\
\hline Transcriptional regulation & $a m t R$ & msmeg_4300 & $\mathrm{n} / \mathrm{a}$ & $\mathrm{n} / \mathrm{a}$ & $\mathrm{n} / \mathrm{a}$ & $\mathrm{n} / \mathrm{a}$ & amtR C. glutamicum [Jakoby et al., 2000] \\
\hline Ammonium uptake & $a m t A$ & msmeg_4635 & $\mathrm{n} / \mathrm{a}$ & $\mathrm{n} / \mathrm{a}$ & $\mathrm{n} / \mathrm{a}$ & $\mathrm{n} / \mathrm{a}$ & Silicibacter pomeroyi (spo_1578) \\
\hline $\begin{array}{l}\text { Assimilatory nitrate } \\
\text { reductase }\end{array}$ & $\begin{array}{l}\text { narI } \\
\text { narJ } \\
\text { narH } \\
\text { narG } \\
\text { narX }\end{array}$ & $\begin{array}{l}\text { msmeg_5137 } \\
\text { msmeg_5138 } \\
\text { msmeg_5139 } \\
\text { msmeg_5140 } \\
n / a\end{array}$ & $\begin{array}{l}R v 1164 \\
R v 1163 \\
R v 1162 \\
R v 1161 \\
R v 1736 c\end{array}$ & $\begin{array}{l}(M L 1499) \\
(M L 1500) \\
(M L 1501) \\
(M L 1502) \\
\mathrm{n} / \mathrm{a}\end{array}$ & $\begin{array}{l}\text { MAP_2617c } \\
M A P \_2618 c \\
M A P \_2619 c \\
M A P \_2620 c \\
\mathrm{n} / \mathrm{a}\end{array}$ & $\begin{array}{l}M b 1196 \\
M b 1195 \\
M b 1194 \\
M b 1193 \\
M b 1765 c\end{array}$ & $\begin{array}{l}\text { [Khan et al., 2008; Sohaskey, 2008; } \\
\text { Sohaskey and Wayne, 2003] }\end{array}$ \\
\hline Nitrite/nitrate transporter & $\begin{array}{l}\text { narK } \\
\text { narK1 } \\
\text { narK2 } \\
\text { narK3 } \\
\text { narU }\end{array}$ & $\begin{array}{l}\text { msmeg_5141 } \\
n / a \\
n / a \\
m s m e g \_0433 \\
n / a\end{array}$ & $\begin{array}{l}\mathrm{n} / \mathrm{a} \\
R v 2329 c \\
R v 1737 c \\
R v 0261 c \\
R v 0267\end{array}$ & $\begin{array}{l}\mathrm{n} / \mathrm{a} \\
\mathrm{n} / \mathrm{a} \\
M L 0844 \\
\mathrm{n} / \mathrm{a} \\
\mathrm{n} / \mathrm{a}\end{array}$ & $\begin{array}{l}\mathrm{n} / \mathrm{a} \\
M A P \_2102 c \\
\mathrm{n} / \mathrm{a} \\
M A P \_3707 c \\
M A P \_3712\end{array}$ & $\begin{array}{l}\mathrm{n} / \mathrm{a} \\
M b 2356 c \\
M b 1766 c \\
M b 0267 c \\
M b 0273\end{array}$ & \\
\hline Unknown & $g \ln A^{*}$ & msmeg_5374 & $\mathrm{n} / \mathrm{a}$ & $\mathrm{n} / \mathrm{a}$ & $\mathrm{n} / \mathrm{a}$ & $\mathrm{n} / \mathrm{a}$ & $\begin{array}{l}\text { Rhodopseudomonas palustris glnA } \\
\text { (RPA0984) }\end{array}$ \\
\hline
\end{tabular}


Table 1 (continued)

\begin{tabular}{|c|c|c|c|c|c|c|c|}
\hline Enzyme/function & $\begin{array}{l}\text { Gene } \\
\text { name }\end{array}$ & $\begin{array}{l}\text { M. smegmatis } \\
\mathrm{mc}^{2} 155\end{array}$ & $\begin{array}{l}\text { M. tuber- } \\
\text { culosis } \\
\text { H37rv }\end{array}$ & $\begin{array}{l}\text { M. leprae } \\
\text { TN }\end{array}$ & $\begin{array}{l}\text { M. avium } \\
\text { ssp. para- } \\
\text { tuberculosis }\end{array}$ & $\begin{array}{l}\text { M. bovis } \\
\text { ssp. bovis } \\
\text { AF2122/97 }\end{array}$ & Reference/nearest homologs \\
\hline Glutamate dehydrogenase & $g d h$ & msmeg_5442 & $\mathrm{n} / \mathrm{a}$ & $\mathrm{n} / \mathrm{a}$ & $\mathrm{n} / \mathrm{a}$ & $\mathrm{n} / \mathrm{a}$ & [Börmann et al., 1992] \\
\hline Ammonium fixation & gltB & msmeg_5594 & $\mathrm{n} / \mathrm{a}$ & $\mathrm{n} / \mathrm{a}$ & $\mathrm{n} / \mathrm{a}$ & $\mathrm{n} / \mathrm{a}$ & $\begin{array}{l}\text { C. glutamicum glutamate synthase } \\
\text { [Beckers et al., 2001; Schulz et al., 2001] }\end{array}$ \\
\hline Transcriptional regulation & $g \ln R$ & msmeg_5784 & $R v 0818$ & $(M L 2194)$ & MAP_0649 & $M b 0841$ & $\begin{array}{l}\text { [Fink et al., 2002; Wray and Fisher, 1993; } \\
\text { Wray et al., 1991] }\end{array}$ \\
\hline Ammonium transporter & $a m t 1$ & msmeg_6259 & $\mathrm{n} / \mathrm{a}$ & $\mathrm{n} / \mathrm{a}$ & $\mathrm{n} / \mathrm{a}$ & $\mathrm{n} / \mathrm{a}$ & Pseudomonas syringae (psyr_2277-2273) \\
\hline \multirow{4}{*}{$\begin{array}{l}\text { Similar to subunits of } \\
\text { glutamate synthetase } \\
\text { and glutamine synthase; } \\
\text { for details, see text }\end{array}$} & $g l x A$ & msmeg_6260 & $\mathrm{n} / \mathrm{a}$ & $\mathrm{n} / \mathrm{a}$ & $\mathrm{n} / \mathrm{a}$ & $\mathrm{n} / \mathrm{a}$ & \\
\hline & $g l x B$ & msmeg_6261 & $\mathrm{n} / \mathrm{a}$ & $\mathrm{n} / \mathrm{a}$ & $\mathrm{n} / \mathrm{a}$ & $\mathrm{n} / \mathrm{a}$ & \\
\hline & $g l x C$ & msmeg_6262 & $\mathrm{n} / \mathrm{a}$ & $\mathrm{n} / \mathrm{a}$ & $\mathrm{n} / \mathrm{a}$ & $\mathrm{n} / \mathrm{a}$ & \\
\hline & $g l x D$ & msmeg_6263 & $\mathrm{n} / \mathrm{a}$ & $\mathrm{n} / \mathrm{a}$ & $\mathrm{n} / \mathrm{a}$ & $\mathrm{n} / \mathrm{a}$ & \\
\hline \multirow[t]{2}{*}{ Ammonium fixation } & gltD & msmeg_6458 & $\mathrm{n} / \mathrm{a}$ & $\mathrm{n} / \mathrm{a}$ & $\mathrm{n} / \mathrm{a}$ & $\mathrm{n} / \mathrm{a}$ & C. glutamicum glutamate synthase \\
\hline & gltB & msmeg_6459 & $\mathrm{n} / \mathrm{a}$ & $\mathrm{n} / \mathrm{a}$ & $\mathrm{n} / \mathrm{a}$ & $\mathrm{n} / \mathrm{a}$ & [Beckers et al., 2001; Schulz et al., 2001] \\
\hline Unknown & $g \ln A^{*}$ & msmeg_6693 & $\mathrm{n} / \mathrm{a}$ & $\mathrm{n} / \mathrm{a}$ & $\mathrm{n} / \mathrm{a}$ & $\mathrm{n} / \mathrm{a}$ & Agrobacterium tumefaciens glnA (Atu4230) \\
\hline
\end{tabular}

activity measurements in $g \ln K$ deletion strains showed that the ATase GlnE works independently from GlnK in S. coelicolor [Hesketh et al., 2002] and C. glutamicum [Strösser et al., 2004].

\section{Transcription Control}

In parallel to the bioinformatics analysis of genes encoding transporters and enzymes related to nitrogen metabolism, a search was carried out for mycobacterial homologs of known transcriptional regulators of nitrogen control in actinomycetes, namely AmtR of C. glutamicum and GlnR of S. coelicolor (table 1). Interestingly, both a homolog to the corynebacterial regulator AmtR (42\% identity) and a protein with high identity to the regulator of streptomycetes, GlnR $(60 \%)$ were found in M. smegmatis, while in all other examined mycobacterial species, only a homolog for GlnR was found (fig. 1). A phylogenetic tree shows that GlnR homologs of pathogenic mycobacteria form a cluster, while $M$. smegmatis GlnR is more closely related to the corresponding N. farcinica and Rhodococcus sp. RHA1 protein (fig. 2a). The M. smegmatis AmtR is more isolated, only AmtR homologs of corynebacteria are clustered and in summary AmtR proteins are less distributed in actinomycetes (fig. 2b).

When all mycobacterial genomes were screened for known cis-acting elements for AmtR and for GlnR, AmtR binding sites could not be identified in any of the mycobacterial genomes. In contrast, using the GlnR motifs of S. coelicolor, Streptomyces avermitilis, and Streptomyces

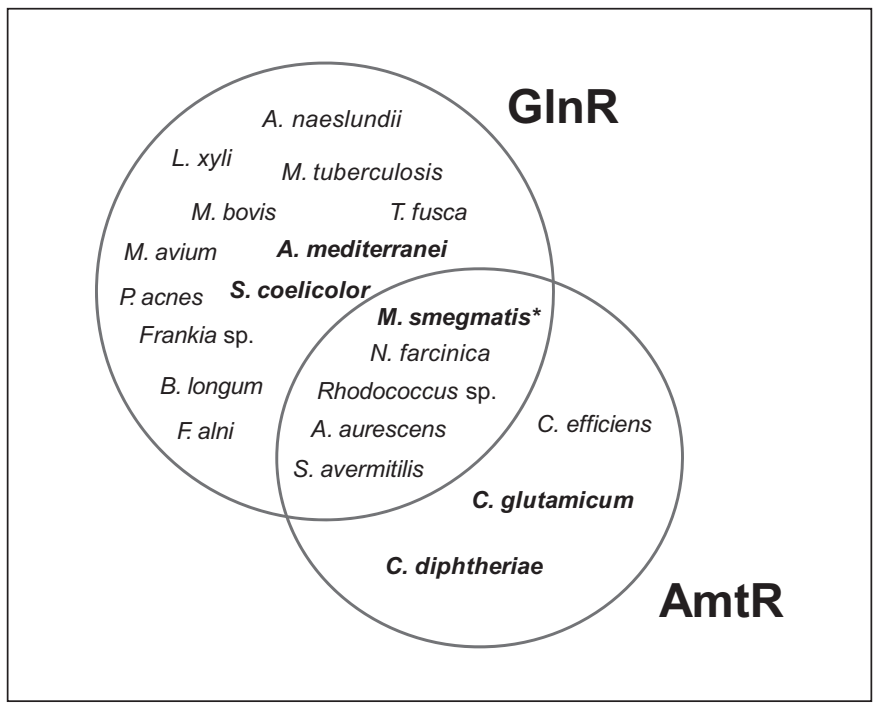

Fig. 1. Distribution of putative nitrogen-dependent transcription regulators GlnR and AmtR in actinomycetes. Bold species names indicate experimental evidence for nitrogen control by the corresponding protein. In $M$. smegmatis (indicated by an asterisk), only the function of GlnR is characterized [Amon et al., 2008], while AmtR function remains unclear [for references of experimentally verified systems see Fink et al., 2002; Jakoby et al., 2000; Nolden et al., 2002; Yu et al., 2006]. Full species names are as follows: Actinomyces naeslundii, Amycolatopsis mediterranei, Arthrobacter aurescens, Bifidobacterium longum, Corynebacterium diphtheriae, Corynebacterium efficiens, Corynebacterium glutamicum, Frankia alni, Leifsonia xyli, Mycobacterium avium, Mycobacterium bovis, Mycobacterium smegmatis, Mycobacterium tuberculosis, Nocardia farcinica, Propionibacterium acnes, Streptomyces avermitilis, Streptomyces coelicolor, and Thermobifida fusca. 


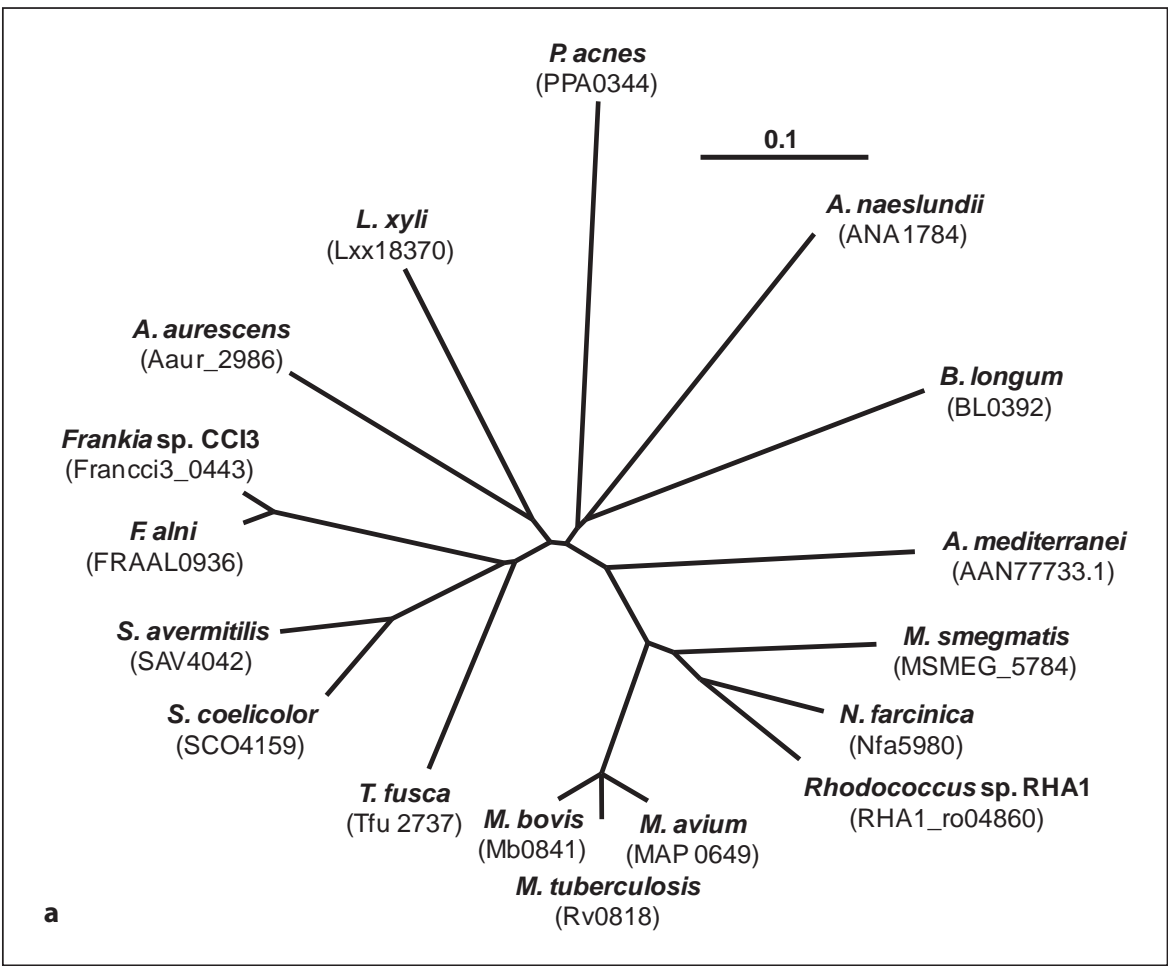

Fig. 2. Phylogenetic trees of the (a) $G \ln R$ and (b) AmtR family of proteins. Unrooted phylogenetic trees were computed with the CLUSTALW software making use of the implemented neighbor joining method with the function for evolutionary distance correction. Evolutionary distances are proportional to the branch length. 17 protein sequences for GlnR and 8 for AmtR homologs were selected as indicated in the figure. For full species names, see figure legend 1.

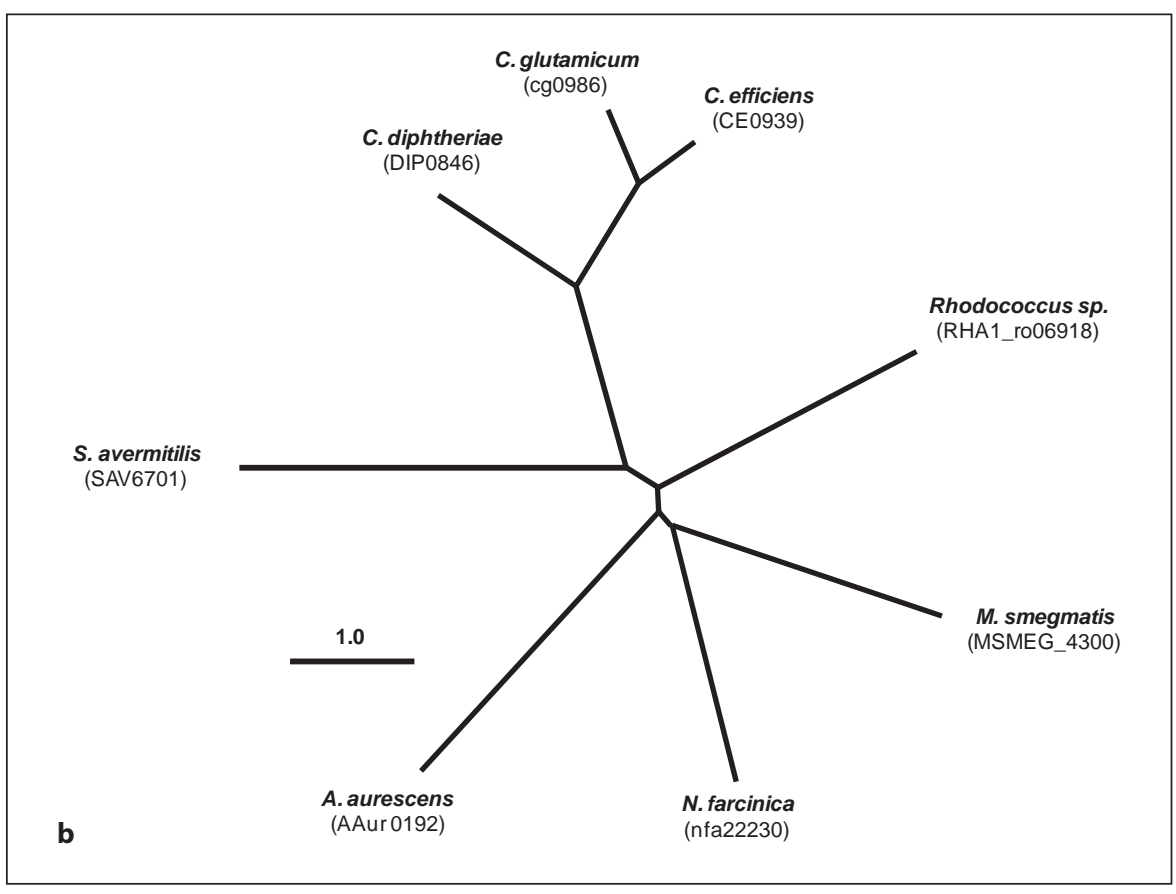

scabies as query sequences, putative binding sites were detected in the available mycobacterial genomes, including three highly conserved cis elements in M. smegmatis (fig. 3). These putative binding motifs were located upstream of the $g \ln A$ gene, coding for glutamine synthetase, as well as upstream of $a m t B$ and $a m t 1$, ammonium permease-encoding genes.

First experimental evidence supports the idea that GlnR is the crucial regulator of nitrogen control in mycobacteria. As shown by in vitro experiments, purified $S$. 
Fig. 3. GlnR-binding motif in mycobacteria. Sequence logo [Crooks et al., 2004] of putative GlnR cis elements identified upstream of the $M$. smegmatis (msmeg), $M$. tuberculosis (H37rv), M. bovis (bovis), and M. avium (avium) gln $A$, amtB, and $a m t 1$ genes (amt1 is exclusively encoded in the M. smegmatis genome).

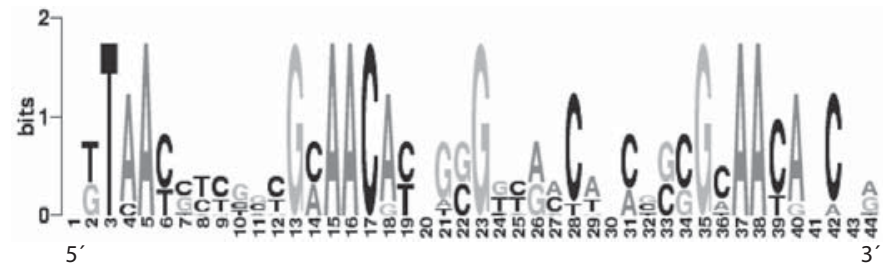

msmeg-glnA H37rv-glnA bovis-glnA avium-glnA msmeg-amt $B$ H37rv-amt B bovis-amt B avium-amt $B$ msmeg-amt 1
CGTAACGTCGGCGCAACATCGGGTTGACGACTGCGCAACATCGT AGTAACGTCTGCGCAACACGGGGTTGACTGACGGGCAATATCGG AGTAACGTCTGCGCAACACGGGGTTGACTGACGGGCAATATCGG CGTAACGTGCGCGCAACATCGGGTTGACTGACGGGCAACATCTG GTTCACTTTCCGGAAACGCAACGGCAGCACCGGCGAAACGCCGG GTTAATCCTGATGAAACATGG GGGCACCATCGCCGCAACAACTA GTTAATCCTGATGAAACATGG GGGCACCATCGCCGCAACAACTA GTTAATCCCGCCGAAACACAG CGGCACTATCGCCGAAACAACCA TTTAACCACGCTGCAACACTTGGCGACCATCTCCGTAACAGAAA coelicolor $\mathrm{GlnR}$ is able to bind to the M. tuberculosis $g \ln A$ promoter region in electrophoretic mobility shift assays [Tiffert et al., 2008]. Furthermore, deletion mutant analyses showed that transcription of $M$. smegmatis amt1, $a m t B, g \ln K, g \ln D$ and $g \ln A$ is controlled by GlnR and that the corresponding mutant strain is unable to respond to nitrogen limitation [Amon et al., 2008].

Comparison of the Nitrogen Metabolism-Related Gene Repertoire of Mycobacteria

M. smegmatis is equipped with a variety of genes enabling the uptake and assimilation of nitrogen sources. Compared to the fast-growing M. smegmatis, all slowgrowing pathogenic members of the genus exhibit a reduced number of genes encoding proteins for nitrogen uptake and assimilation (see table 1 for details and fig. 4 for comparison of M. smegmatis and M. tuberculosis). This is also due to the fact that M. smegmatis seems to have acquired an astonishingly wide range of nitrogenrelated genes and gene regions via horizontal gene transfer from a variety of other bacteria, such as Agrobacterium, Burkholderia, and Pseudomonas species. This includes among others a second urease operon, additional ammonium transporters, and a broad variety of glutamine synthetases of various classes and origins (table 1). According to the genomic data, M. smegmatis is capable of the active uptake and assimilation of a comparatively wide range of substrates for the extraction of ammonium and further assimilation into central metabolites such as glutamate and glutamine, which is in good concordance to the situation found for the uptake and assimilation of carbohydrates in M. smegmatis [Titgemeyer et al., 2007] and thus exhibits a similar repertoire of nitrogen-related genes to that of C. glutamicum [Burkovski, 2007; Hänssler and Burkovski, 2008]. Another interesting fact is the cooccurrence of homologs of both regulators of the nitrogen metabolism in actinomycetes in the genome of $M$. smegmatis, namely AmtR and GlnR. While the transcriptional repressor AmtR is the global regulator of nitrogen metabolism in corynebacteria [Walter et al., 2007], this function was only recently shown for the M. smegmatis $\mathrm{GlnR}$ and its respective target genes amt1, amtB, and $g \ln A$ [Amon et al., 2008]; as only homologs of GlnR are found in the genomes of other mycobacteria (fig. 1), the role of AmtR for M. smegmatis remains to be further explored.

All mycobacteria investigated exhibit the subunits for a respiratory nitrate reductase (which are nonfunctional pseudogenes in $M$. leprae), while especially the tuberculoid members possess multiple homologs of the E. coli nitrite/nitrate antiporters, NarK and NarU. For M. tuberculosis it has already been shown that nitrate respiration plays an important role during hypoxia [Sohaskey, 2008], but the additional occurrence of a nitrite reductase, besides its role in detoxification by reduction of nitrite, points to a possible involvement of the enzyme in the complete reduction of nitrate to ammonium and following assimilation, which has been demonstrated for $M$. smegmatis [Khan et al., 2008].

M. leprae unsurprisingly reveals the strongest reduction of nitrogen metabolism-related genes as a process of gene decay termed 'reductive evolution' [Gómez-Valero 
Fig. 4. Comparison of proteins involved in nitrogen uptake, metabolism and control in (a) M. smegmatis and (b) M. tuberculosis. Given are the protein names of the respective transporters and enzymes as derived from the in silico analyses (for details, see text). Question marks indicate putative protein-protein interactions and unknown signals. Multiple copies of enzymes are indicated by asterisks.
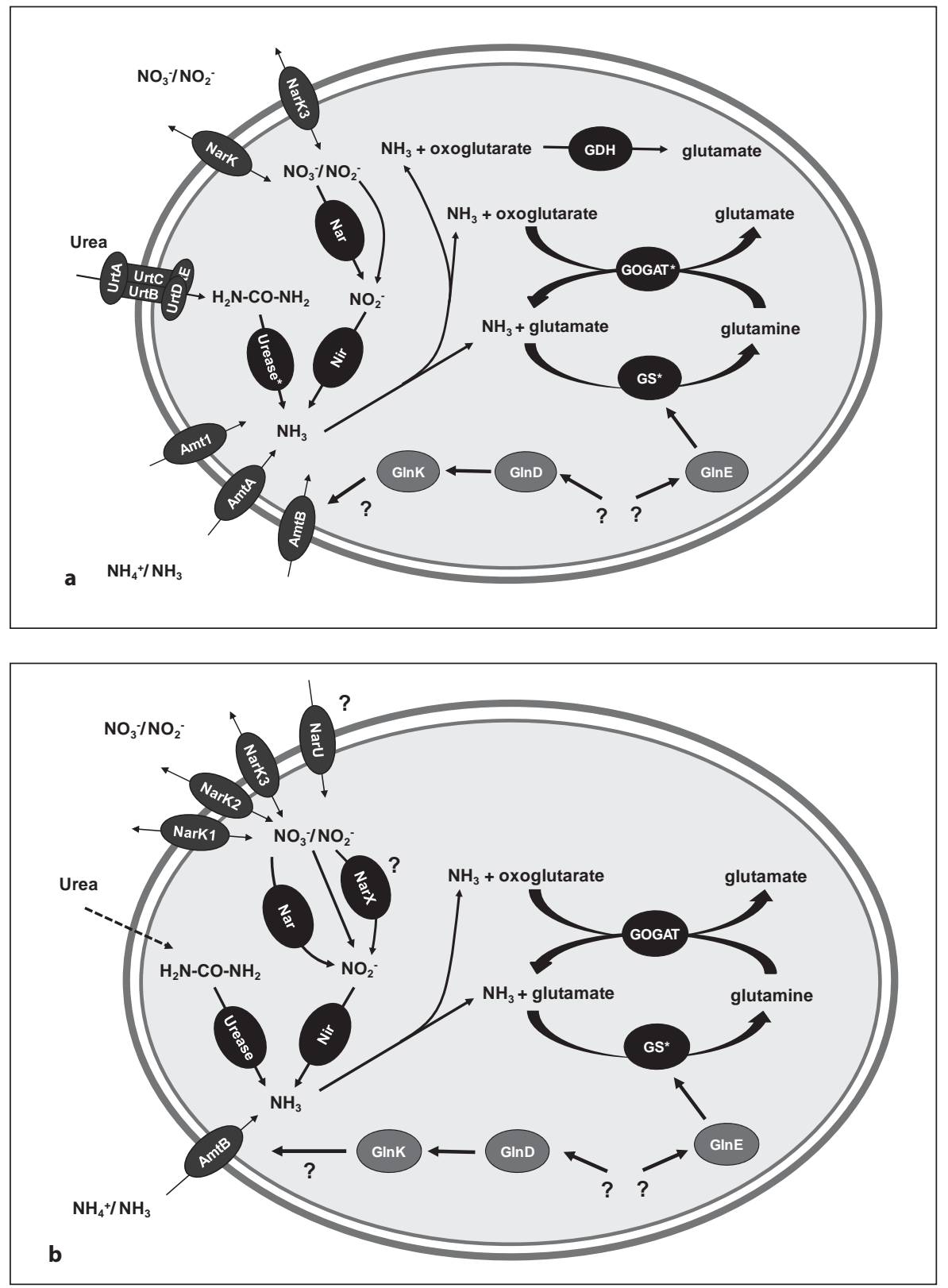

et al., 2007], resulting in a minimal set of genes required for a functional nitrogen metabolism. This set comprises the genes of the GS/GOGAT pathway $(g l t B D, g \ln A)$ as well as the GS ATase $(g \ln E)$. While we did not find any nitrogen-specific transport systems besides one nitrite/ nitrate antiporter (NarK1), the genome of M. leprae features putative amino acid and oligopeptide transporters and permeases (data not shown); these substrates could very well represent the main nitrogen sources, taking into account the lifestyle of $M$. leprae as an obligate in- tracellular parasite [Sassetti et al., 2003; Vissa and Brennan, 2001].

\section{Concluding Remarks}

A growing number of genome sequences of different actinomycetes and useful tools and databases for whole genome screening and comparison are already available for research. Due to different studies based on these databases in combination with experimental approaches the transcriptional regulation of nitrogen control in acti- 
nomycetes is now well understood. However, there are still a number of open questions regarding the post-translational interactions and modifications between the key players of nitrogen control (fig. 4). For example, the sensory protein for the nitrogen status of the cell that putatively controls the GlnR regulator as well as the signal sensed by this protein is still unknown, and also the function of the $\mathrm{P}_{\mathrm{II}}$-type signal transduction proteins is unclear. Furthermore, the existence of two different nitrogen-dependent transcription regulators in the actinomycetes, namely GlnR and AmtR, is still a mystery. The here compiled overview of nitrogen-related genes might thus serve as a blueprint to direct future studies.

\section{Experimental Procedures}

In silico Analysis of Mycobacterial Genome Repertoire

A bioinformatic screen of the available genome sequences of M. smegmatis, M. tuberculosis, M. leprae, M. avium, and M. bovis was performed. For prediction of genes encoding proteins involved in uptake and assimilation of nitrogen sources as well as in nitrogen signal transduction and nitrogen control, genome data obtained from The Institute for Genomic Research (http:// www.tigr.org) were screened. To find the most representative mycobacterial homologs of B. subtilis, E. coli, C. glutamicum and $S$. coelicolor proteins, we used single genome protein and nucleotide BLAST available at the following genome server sites: http://genolist.pasteur.fr, TigrBLAST (http://tigrblast.tigr.org/ cmr-blast/), CoryneRegNet (http://www.cebitec.uni-bielefeld.de [Baumbach, 2007]) and ScoDB (http://streptomyces.org.uk). Prediction of the possible function was based on the following crite- ria: (1) protein identity of the mycobacterial protein of more than $30 \%$ to the homologous protein of E. coli or B. subtilis, more than $50 \%$ to S. coelicolor or C. glutamicum, or more than $60 \%$ to other mycobacterial proteins, (2) when more than one gene of the corresponding operon was conserved, and (3) most importantly, a solid biochemical analysis of the homologous protein was available. Sequence alignments were conducted with CLUSTALW applying pre-defined algorithms of The European Bioinformatics Institute (EBI) at The European Molecular Biology Laboratory (EMBL) (http://www.ebi.ac.uk/clustalw). Phylogenetic trees were calculated with the neighbour joining method as implemented in CLUSTALW and graphically visualized by importing phylip (*.ph) files into the TREEVIEW software (http://taxonomy.zoology.gla.ac.uk/rod/treeview.hmtl).

Screening for Putative Transcriptional Regulator Binding Sites All mycobacterial genomes were screened for conserved cis elements using the already published binding motifs of the corynebacterial AmtR [Beckers et al., 2005; Walter et al., 2007] and the Streptomyces GlnR [Reuther and Wohlleben, 2007] as query sequences using the PreDetector program [Hiard et al., 2007]. The screening for putative cis elements was performed by first creating positional weight matrices with different lengths (in bp) for the known cis elements of AmtR and GlnR with the Weight Matrix Creation module of PreDetector, then using these weight matrices in the Regulon Prediction module with the genomes of interest. All genomes to be examined were obtained automatically from the NCBI GenBank and downloaded to hard disk for later use.

\section{Acknowledgements}

The authors were supported by the Deutsche Forschungsgemeinschaft (SFB473, C12 and D4).

\section{References}

Amon J, Bräu T, Grimrath A, Hänßler E, Hasselt K, Höller M, Jeßberger N, Ott L, Szököl J, Titgemeyer F, Burkovski A: Nitrogen control in Mycobacterium smegmatis: Nitrogen-dependent expression of ammonium transport and assimilation proteins depends on OmpRtype regulator GlnR. J Bacteriol 2008, Epub ahead of print.

Bange FC, Collins FM, Jacobs WR Jr: Survival of mice infected with Mycobacterium smegmatis containing large DNA fragments from Mycobacterium tuberculosis. Tuber Lung Dis 1999;79:171-180.

Baumbach J: CoryneRegNet 4.0 - A reference database for corynebacterial gene regulatory networks. BMC Bioinformatics 2007;8:429.

-Beckers G, Nolden L, Burkovski A: Glutamate synthase of Corynebacterium glutamicum is not essential for glutamate synthesis and is regulated by the nitrogen status. Microbiology 2001;147:2961-2970.
Beckers G, Bendt AK, Krämer R, Burkovski A: Molecular identification of the urea uptake system and transcriptional analysis of urea transporter- and urease-encoding genes in Corynebacterium glutamicum. J Bacteriol 2004; 186:7645-7652.

Beckers G, Strösser J, Hildebrandt U, Kalinowski J, Farwick M, Krämer R, Burkovski A: Regulation of AmtR-controlled gene expression in Corynebacterium glutamicum: mechanism and characterization of the AmtR regulon. Mol Microbiol 2005;58:580-595.

Börmann ER, Eikmanns BJ, Sahm H: Molecular analysis of the Corynebacterium glutamicum gdh gene encoding glutamate dehydrogenase. Mol Microbiol 1992;6:317-326.

Burkovski A: I do it my way: Regulation of ammonium uptake and ammonium assimilation in Corynebacterium glutamicum. Arch Microbiol 2003a;179:83-88.
Burkovski A: Ammonium assimilation and nitrogen control in Corynebacterium glutamicum and its relatives: an example for new regulatory mechanisms in actinomycetes. FEMS Microbiol Rev 2003b:27:617-628.

Burkovski A: Nitrogen metabolism and its regulation; in Bott M, Eggeling L (eds): Handbook of Corynebacterium glutamicum. Boca Raton, CRC Press LLC, 2005, pp 333-349.

Burkovski A: Nitrogen control in Corynebacterium glutamicum: proteins, mechanisms, signals. J Microbiol Biotechnol 2007;17:187194.

Carroll P, Pashley CA, Parish T: Functional analysis of GlnE, an essential adenylyl transferase in Mycobacterium tuberculosis. J Bacteriol 2008;DOI:10.1128/JB.00166-08.

Clemens DL, Lee BY, Horwitz MA: Purification, characterization, and genetic analysis of $M y$ cobacterium tuberculosis urease, a potentially critical determinant of host-pathogen interaction. J Bacteriol 1995;177:5644-5652. 
- Cole ST, Brosch R, Parkhill J, Garnier T, Churcher C, Harris D, et al: Deciphering the biology of Mycobacterium tuberculosis from the complete genome sequence. Nature 1998; 393:537-544

-Cole ST, Eiglmeier K, Parkhill J, James KD, Thomson NR, Wheeler PR, et al: Massive gene decay in the leprosy bacillus. Nature 2001;409:1007-1011.

-Crooks GE, Hon G, Chandonia JM, Brenner SE: WebLogo: a sequence logo generator. Genome Res 2004;14:1188-1190.

Detsch C, Stülke J: Ammonium utilization in Bacillus subtilis: transport and regulatory functions of NrgA and NrgB. Microbiology 2003;149:3289-3297.

Dye C, Watt CJ, Bleed DM, Hosseini SM, Raviglione MC: Evolution of tuberculosis control and prospects for reducing tuberculosis incidence, prevalence, and deaths globally. JAMA 2005;293:2767-2775.

Fink D, Weisschuh N, Reuther J, Wohlleben W, Engels A: Two transcriptional regulators GlnR and GlnRII are involved in regulation of nitrogen metabolism in Streptomyces coelicolor A3(2). Mol Microbiol 2002;46:331347.

Garnier T, Eiglmeier K, Camus JC, Medina N, Mansoor H, Pryor M, et al: The complete genome sequence of Mycobacterium bovis. Proc Natl Acad Sci USA 2003;100:78777882 .

-Gómez-Valero L, Rocha EPC, Latorre A, Silva FJ: Reconstructing the ancestor of Mycobacterium leprae: The dynamics of gene loss and genome reduction. Genome Res 2007;17: 1178-1185.

Hänßler E, Burkovski A: Molecular mechanisms of nitrogen control in corynebacteria; in Burkovski A (ed): Corynebacteria: Genomics and Molecular Biology. Wymondham, Caister Academic Press, 2008, pp 183-201.

-Harth G, Clemens DL, Horwitz AA: Glutamine synthetase of Mycobacterium tuberculosis: extracellular release and characterization of its enzymatic activity. Proc Natl Acad Sci USA 1994;91:9342-9346.

-Harth G, Maslesa-Galic S, Tullius MV, Horwitz AA: All four Mycobacterium tuberculosis $\operatorname{gln} A$ genes encode glutamine synthetase activities but only GlnA1 is abundantly expressed and essential for bacterial homeostasis. Mol Microbiol 2005;58:1157-1172.

-Hesketh A, Fink D, Gust B, Rexer H-U, Scheel B, Chater K, Wohlleben W, Engels A: The GlnD and GlnK homologues of Streptomyces coelicolor A3(2) are functionally dissimilar to their nitrogen regulatory system counterparts from enteric bacteria. Mol Microbiol 2002;46:319-330.

- Hiard S, Maree R, Colson S, Hoskisson PA, Titgemeyer F, van Wezel G, Joris B, Wehenkel L, Rigali S: PREDetector: a new tool to identify regulatory elements in bacterial genomes. Biochem Biophys Res Commun 2007;357:861-864.
Hotter GS, Mouat P, Collins DM: Independent transcription of glutamine synthetase $(g \ln A 2)$ and glutamine synthetase adenylyltransferase $(g \ln E)$ in Mycobacterium bovis and Mycobacterium tuberculosis. Tuberculosis DOI:10.1016/j.tube.2008.02.006.

Jakoby M, Krämer R, Burkovski A: Nitrogen regulation in Corynebacterium glutamicum: Isolation of genes involved and biochemical characterization of the corresponding proteins. FEMS Microbiol Lett 1999;173:303310.

Jakoby M, Nolden L, Meier-Wagner J, Krämer R, Burkovski A: AmtR, a global repressor in the nitrogen regulation system of Corynebacterium glutamicum. Mol Microbiol 2000;37: 964-977.

Khan A, Akhtar S, Ahmad J, Sarkar D: Presence of a functional nitrate pathway in Mycobacterium smegmatis. Microb Pathog 2008;44: 71-77.

Li L, Bannantine JP, Zhang Q, Amonsin A, Max BJ, Alt D, Banerji N, Kanjilal S, Kapur V: The complete genome sequence of Mycobacterium avium subspecies paratuberculosis. Proc Natl Acad Sci USA 2005;102:12344-12349.

-Meier-Wagner J, Nolden L, Jakoby M, Siewe R, Krämer R, Burkovski A: Multiplicity of ammonium uptake systems in Corynebacterium glutamicum: role of Amt and AmtB. Microbiology 2001;147:135-143.

- Nolden L, Beckers G, Burkovski A: Nitrogen assimilation in Corynebacterium diphtheriae: pathways and regulatory cascades. FEMS Microbiol Lett 2002;208:287-293.

-Nordqvist A, Nilsson MT, Röttger S, Odell LR, Krajewski WW, Andersson CE, Larhed M, Mowbray SL, Karlén A: Evaluation of the amino acid binding site of Mycobacterium tuberculosis glutamine synthetase for drug discovery. Bioorg Med Chem 2008;6:5501-5513.

- Parish T, Stoker NG: $g \ln E$ is an essential gene in Mycobacterium tuberculosis. J Bacteriol 2000;182:5715-5720.

- Pashley CA, Brown AC, Robertson D, Parish T: Identification of the Mycobacterium tuberculosis $\ln E$ promoter and its response to nitrogen availability. Microbiology 2006;152: 2727-2734.

Pelicic V, Reyrat JM, Gicquel B: Genetic advances for studying Mycobacterium tuberculosis pathogenicity. Mol Microbiol 1998;28:413420.

Read R, Pashley CA, Smith D, Parish T: The role of GlnD in ammonium assimilation in $M y$ cobacterium tuberculosis. Tuberculosis 2007 ; 87:384-390.

Reuther J, Wohlleben W: Nitrogen metabolism in Streptomyces coelicolor: Transcriptional and post-translational regulation. J Mol Microbiol Biotechnol 2007;12:139-146.

- Rexer HU, Schäberle T, Wohlleben W, Engels A: Investigation of the functional properties and regulation of three glutamine synthetase-like genes in Streptomyces coelicolor A3(2). Arch Microbiol 2006;186:447-458.

- Sassetti CM, Boyd DH, Rubin EJ: Genes required for mycobacterial growth defined by high density mutagenesis. Mol Microbiol 2003;48: $77-84$.
-Schulz AA, Collett HJ, Reid SJ: Regulation of glutamine synthetase and glutamate synthase in Corynebacterium glutamicum ATCC 13032. FEMS Microbiol Lett 2001;205:361-367.

Sohaskey CD: Nitrate enhances the survival of Mycobacterium tuberculosis during inhibition of respiration. J Bacteriol 2008;190: 2981-2986.

Sohaskey CD, Wayne LG: Role of narK2X and narGHJI in hypoxic upregulation of nitrate reduction by Mycobacterium tuberculosis. J Bacteriol 2003; 185:7247-7256.

-Strösser J, Lüdke A, Schaffer S, Krämer R, Burkovski A: Regulation of GlnK activity: modification, membrane sequestration, and proteolysis as regulatory principles in the network of nitrogen control in Corynebacterium glutamicum. Mol Microbiol 2004;54: 132-147.

Tiffert Y, Supra P, Wurm R, Wohlleben W, Wagner R, Reuther J: The Streptomyces coelicolor GlnR regulon: identification of new $G \ln R$ targets and evidence for a central role of GlnR in nitrogen metabolism in actinomycetes. Mol Microbiol 2008;67:436-446.

- Titgemeyer F, Parche S, Mahfoud M, Amon J, Bail J, Schlicht M, Hillmann D, Rehm N, Stephan J, Walter B, Burkovski A, Niederweis M: Sugar transport in Mycobacterium smegmatis and Mycobacterium tuberculosis. J Bacteriol 2007;189:5903-5915.

- Tullius MV, Harth G, Horwitz AA: Glutamine synthetase $\mathrm{Gln} A 1$ is essential for growth of Mycobacterium tuberculosis in human THP1 macrophages and guinea pigs. Infect Immun 2003;71:3927-3936.

-Vissa VD, Brennan PJ: The genome of Mycobacterium leprae: a minimal mycobacterial genome set. Genome Biol 2001;2:1023.11023.8.

-Walter B, Hänßler E, Kalinowski J, Burkovski A: Nitrogen metabolism and nitrogen control in corynebacteria: variations of a common theme. J Mol Microbiol Biotechnol 2007;12: 131-138.

-Walter B, Küspert M, Ansorge A, Krämer R, Burkovski A: Dissection of ammonium uptake systems in Corynebacterium glutamicum: mechanism and energetics of AmtA and AmtB. J Bacteriol 2008;190:2611-2614.

Wang H, Gunsalus RP: The nrfA and nirB nitrite reductase operons in Escherichia coli are expressed differently in response to nitrate than to nitrite. J Bacteriol 2000;182:5813-5822.

Wray LV, Atkinson MR, Fisher SH: Identification and cloning of the $g \ln R$ locus, which is required for transcription of the $g \ln A$ gene in Streptomyces coelicolor A3(2). J Bacteriol 1991;173:7351-7360.

-Wray LV, Fisher SH: The Streptomyces coelicolor $g \ln R$ gene encodes a protein similar to other bacterial response regulators. Gene 1993; 130:145-150

Yu H, Peng W, Liu Y, Wu T, Yao Y, Cui M, Jiang W, Zhao G-P: Identification and characterization of $\ln A$ promoter and its corresponding trans-regulating protein $\mathrm{GlnR}$ in the rifamycin SV producing actinomycete, Amycolatopsis mediterranei U32. Acta Biochim Biophys Sin 2006;38:831-843. 